\section{(1) \\ CrossMark}

\title{
Minimal clinically important difference for asthma endpoints: an expert consensus report
}

\author{
Matteo Bonini, ${ }^{1,2}$, Marcello Di Paolo (1) 1 , Diego Bagnasco ${ }^{3}$, Ilaria Baiardini ${ }^{4}$, \\ Fulvio Braido ${ }^{3}$, Marco Caminati $^{5,6}$, Elisiana Carpagnano ${ }^{7}$, Marco Contoli $^{8}$, \\ Angelo Corsico 9 , Stefano Del Giacco (10 ${ }^{10}$, Enrico Heffler ${ }^{11}$, Carlo Lombardi ${ }^{12}$, \\ Ilaria Menichini ${ }^{13}$, Manlio Milanese ${ }^{14}$, Nicola Scichilone (ib) \\ Gianenrico Senna ${ }^{5,6}$ and Giorgio W. Canonica ${ }^{11}$
}

\begin{abstract}
Affiliations: ${ }^{1}$ National Heart and Lung Institute, Royal Brompton Hospital \& Imperial College London, London, UK. ${ }^{2}$ UOC Pneumologia, Istituto di Medicina Interna, F. Policlinico Gemelli IRCCS, Università Cattolica del Sacro Cuore, Rome, Italy. ${ }^{3}$ Allergy and Respiratory diseases, University of Genoa, Dept of Internal Medicine (DiMI), Ospedale Policlinico San Martino, Genoa, Italy. ${ }^{4}$ Dept of Biomedical Sciences, Humanitas University, Pieve Emanuele, Italy. ${ }^{5}$ Dept of Medicine, University of Verona, Verona, Italy. ${ }^{6}$ Asthma Center and Allergy Unit, Verona University Hospital, Verona, Italy. ${ }^{7}$ Division of Respiratory Diseases, Dept of Medical and Surgical Sciences, University of Foggia, Italy. ${ }^{8}$ Section of Internal and Cardiorespiratory Medicine, Dept of Medical Sciences, University of Ferrara, Ferrara, Italy. ${ }^{9}$ Division of Respiratory Diseases, IRCCS Policlinico San Matteo Foundation - Dept of Internal Medicine and Therapeutics, University of Pavia, Pavia, Italy. ${ }^{10}$ Dept of Medical Sciences and Public Health, University of Cagliari, Cagliari, Italy. ${ }^{11}$ Personalized Medicine, Asthma and Allergy - Humanitas Research Hospital, Rozzano, Italy. ${ }^{12}$ Departmental Unit of Pneumology \& Allergology, Istituto Ospedaliero Fondazione Poliambulanza, Brescia, Italy. ${ }^{13}$ Dept of Cardiovascular, Respiratory, Nephrology, Anaesthesiology and Geriatric Science, "Sapienza" University, Rome, Italy. ${ }^{14}$ Division of Pulmonology, S Corona Hospital, Savona, Italy. ${ }^{15}$ AOUP Policlinico Universitario, DIBIMIS, Università di Palermo, Palermo, Italy.
\end{abstract}

Correspondence: Marco Caminati, Dept of Medicine, University of Verona, Piazzale Stefani 1, 37126 Verona, Italy. E-mail: ma.caminatiagmail.com

@ERSpublications

Minimal clinically important difference (MCID) cut-offs in asthma are validated for patient reported outcomes and lung function, but not for inflammatory biomarkers. MCID represents a key target for future development in asthma management. http://bit.ly/33hcWIe

Cite this article as: Bonini M, Di Paolo M, Bagnasco D, et al. Minimal clinically important difference for asthma endpoints: an expert consensus report. Eur Respir Rev 2020; 29: 190137 [https://doi.org/10.1183/ 16000617.0137-2019].

ABSTRACT Minimal clinically important difference (MCID) can be defined as the smallest change or difference in an outcome measure that is perceived as beneficial and would lead to a change in the patient's medical management. The aim of the current expert consensus report is to provide a "state-of-the-art" review of the currently available literature evidence about MCID for end-points to monitor asthma control, in order to facilitate optimal disease management and identify unmet needs in the field to guide future research.

A series of MCID cut-offs are currently available in literature and validated among populations of asthmatic patients, with most of the evidence focusing on outcomes as patient reported outcomes, lung function and exercise tolerance. On the contrary, only scant and partial data are available for inflammatory biomarkers. These clearly represent the most interesting target for future development in diagnosis and clinical management of asthma, particularly in view of the several biologic drugs in the pipeline, for which regulatory agencies will soon require personalised proof of efficacy and treatment response predictors.

Provenance: Submitted article, peer reviewed

Received: 25 Oct 2019 | Accepted after revision: 09 March 2020

Copyright $\odot$ ERS 2020. This article is open access and distributed under the terms of the Creative Commons Attribution Non-Commercial Licence 4.0. 


\section{Introduction}

Asthma is a heterogeneous chronic respiratory disease which affects approximately 300 million people worldwide across ages and ethnic groups [1], with an additional 100 million subjects estimated to be asthmatic by 2025 [2]. Therefore, asthma represents a relevant socioeconomic burden in terms of mortality, morbidity, quality of life and healthcare costs, in both adults and children [3].

It has been recommended that optimal asthma management should primarily aim to achieve and maintain disease control [4-7]. The concept of asthma control is central to all current asthma guidelines and its degree should be assessed taking into consideration several parameters, including clinical, functional and inflammatory end-points, as well as patient reported outcomes (PROs). However, with this regard, there is often a lack of consensus about the target value or the change it should be aimed towards.

Quantifying statistical significance of evidence by using Fisher's p-value in the context of null hypothesis testing represents one of the most widely used methods to guide decision making in medical research. However, although the p-value often objectifies research outcomes, the statistical significance does not necessarily imply clinical relevance. It is therefore vital for healthcare providers to bridge the gap between statistical and clinical significance both in daily practice and clinical trials in order to achieve an adequately informed decision in recommending interventions. Hence, the importance of determining the minimal clinically important difference (MCID).

MCID was first described in 1989 by JAESCHKE et al. [8] and can be defined as the smallest change or difference in an outcome measure that is perceived as beneficial and would lead to a change in the patient's medical management, assuming an absence of excessive side-effects and costs. This is based on the consideration that identical changes on a numerical scale may have diverse clinical importance in different subjects and populations.

The aim of the current expert consensus report is to provide a "state-of-the-art" review of the currently available evidence about MCID for end-points to monitor asthma control, in order to facilitate an optimal disease management and identify unmet needs in the field to guide future research.

Electronic searches were undertaken in Medline, Web of Science (ISI), SCOPUS and the Cochrane Library (CENTRAL) up to 2019 by combining the following keywords: "asthma", "endpoint", "outcome", "minimal clinically important difference" and "MCID".

\section{How to determine the MCID}

MCID represents the best standard for determining effectiveness of a given treatment and describes patient satisfaction regarding a given intervention. A number of approaches have been proposed for establishing MCID, mainly clustered in three broad categories: consensus-, distribution- and anchor-based methods [9-14].

\section{Consensus-based method}

Also known as the Delphi method, this is based on the opinion of the experts on which numerical value should represent a clinically relevant change for the considered end-point. These assessments are frequently revised until consensus is reached.

\section{Distribution-based methods}

These methods rely on the distribution of observed scores in specific populations of patients and reflect one or more statistical indices of change. They allow the magnitude of change to be described. Several distribution-based methods are available to determine MCID (table 1) [15-21], with a main advantage for being easy to calculate. Despite, primarily for such reasons, distribution-based methods being widely used in scientific publications as an estimate or evidence of clinical significance, they present some significant limitations. Most importantly they provide a minimal value below which a change in outcome scores for a given measure may be due to a measurement error. Thus, these methods neglect the core concept of MCID, which is to determine the clinical importance of a given change in outcome scores independently from their statistical significance [22]. These are the less "patient-centred" methods, and therefore should not be used as a first choice to determine MCID.

\section{Anchor-based methods}

These methods consist in linking a specific change in the outcome measure score to a meaningful external indicator (i.e. the anchor), either clinical or patient reported. They are adopted to classify individuals into groups according to the degree and direction of change. The anchor accounts for patient's perspective; for example, the perception of benefit from the treatment, and allows to assign a clinical meaning to the assessed change. The most widely used external criterion in the anchor-based approach is the global rating of change (GRC). This consists in a Likert-type scale scored by the patient [22], from "large deterioration" through "no 
TABLE 1 Distribution-based measures suggested to determine minimal clinically important difference (MCID)

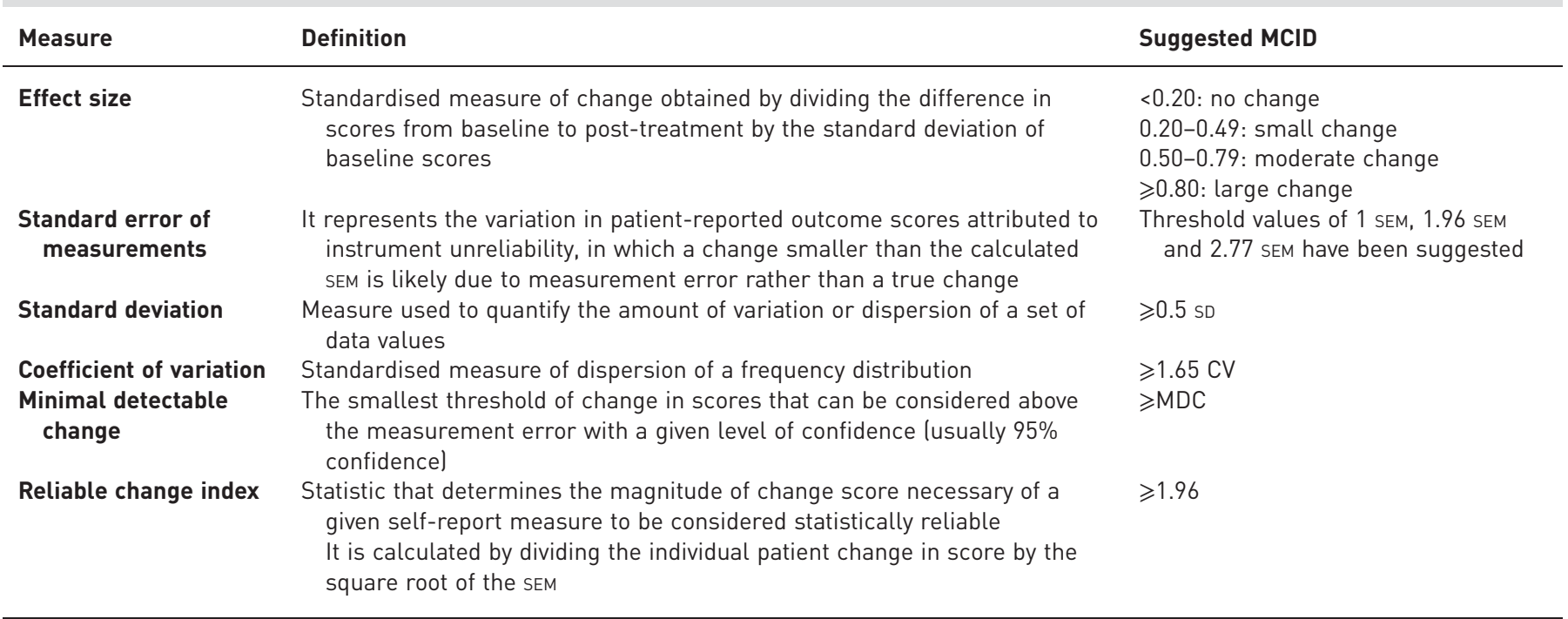

change" to "large improvement". The original statistical strategy to determine MCID using the GRC was based on the mean change of patients rating themselves as having a small improvement or deterioration. However, more recently it has been recognised that the precision of MCID can be optimised by evaluating the entire cohort of patients with receiver operating characteristics curve analyses rather than with two groups adjacent to the dichotomisation point [23]. The comprehensive review written by CROSBY et al. [24] provides a detailed description of the various anchor-based approaches and recommend longitudinal methods as more reliable, compared to cross-sectional ones, to determine an individual change. Anchor-based methods have the advantage of linking the change in a given score to the patient's perspective and provide insights on the importance of observed change form a subjective point of view. However, anchor-based methods do not take into account the measurement precision of the instrument. Consequently, these methods do not provide any information about the range of change that would be expected by random variation alone [24]. Moreover, it has underlined the paradox to use a subjective measure, as a supposed external criterion, for another subjective measure of the same or similar construct [12]. When assessing the same construct, it is therefore essential to use two independent measurements [25].

In summary, each of these methods has its own limitations. In the Delphi method, expert opinion could not coincide with patients' feeling. Anchor-based methods are limited by the choice of an anchor, which is subjective and difficult to find it valid and reliable. Additionally, it might happen that patients fail to understand the context of improvement or to remember the intrinsic nature of their prior condition. Finally, distribution-based methods, relying on purely statistical estimates, can only identify minimal detectable effects and prevent the definition of the clinical importance of a given change in the lack of consensus.

Hence, it has been suggested that the best strategy to determine MCID should be based on a multiple approach, assigning the highest relative weight to anchor-based methods and using distribution-based measures as supportive information [26].

\section{Functional end-points \\ Proximal airways parameters}

Clinical trials in asthma often include forced expiratory volume in $1 \mathrm{~s}\left(\mathrm{FEV}_{1}\right)$ as a primary outcome, mainly because the research community and regulatory agencies have traditionally recognised its importance as an objective index of airflow obstruction [27]. Peak expiratory flow also reflects airway obstruction and is often considered as one of the main end-points in the evaluation of the efficacy of anti-asthma treatment. Other spirometric measures, such as vital capacity and forced vital capacity (FVC) have been used as complementary end-points in asthma trials.

Several attempts to identify MCIDs in lung function tests by the use of a distribution-based method have been carried out, even if the great majority of the available evidence originated from studies with patients affected by COPD. PENNOCK et al. [28], assuming a within-a-day coefficient of variation of $6.7 \%$ for $\mathrm{FEV}_{1}$ and $8.1 \%$ for FVC in a population of subjects with reversible airflow obstruction, estimated thresholds for acute significant changes in $\mathrm{FEV}_{1}$ and $\mathrm{FVC}$ of $11 \%$ and $13 \%$, respectively. The largest amount of data 
about reproducibility of spirometry from week to week was reported from the Lung Health Study [29], where spirometry was repeated after 17 days in 5885 subjects with mild obstructive disease. In this population, coefficient of variation for $\mathrm{FEV}_{1}$ was $4.1-4.9 \%$. Higher variability coefficients for $\mathrm{FEV}_{1}$ and FVC have been reported when the tests are separated by a greater period of time (years) [30]. According to the latest American Thoracic Society/European Respiratory Society recommendations for the interpretation of lung function tests, changes in $\mathrm{FEV}_{1} \geqslant 20 \%$ in short-term trials (i.e. weeks of duration) and $\geqslant 15 \%$ in long-term trials (i.e. $\geqslant 1$ year) were reported to be confident that a clinically meaningful change had occurred [30].

Airflow obstruction is also evaluated by the $\mathrm{FEV}_{1}$ to $\mathrm{FVC}$ ratio $\left(\mathrm{FEV}_{1} / \mathrm{FVC}\right)$, which is considered to be abnormal if below the 5 th percentile of the frequency distribution of values measured in healthy people of comparable sex and age [30]. The use of a fixed cut-off for $\mathrm{FEV}_{1} / \mathrm{FVC}($ e.g. <0.7) could lead to underestimation or overestimation of airflow obstruction in young and elderly asthmatic patients, respectively, providing a misleading assessment [31].

There is no evidence of a MCID for the change in $\mathrm{FEV}_{1} / \mathrm{FVC}$ but the normalisation of the ratio, secondary to an improvement in $\mathrm{FEV}_{1}$, could be considered clinically important.

Airway hyperresponsiveness, defined as an increased sensitivity and exaggerated response to non-allergenic provocation agents (such as histamine or methacholine), and post-bronchodilator reversibility test, positive when an increase in $\mathrm{FEV}_{1}$ and/or FVC $>12 \%$ and $200 \mathrm{~mL}$ compared to baseline [27, 30] is observed, are both recommended for asthma diagnosis and in specific conditions for asthma control monitoring. A specific MCID related to their improvement has not been investigated so far. However, by analysing a cohort of 302 asthmatics, PEREZ et al. [32] identified $\mathrm{FEV}_{1}$ reversibility as the only parameter associated with a significant clinical improvement (change in visual analogue scale $\geqslant 2 \mathrm{~cm}$ ) in terms of dyspnoea or chest tightness.

\section{Peripheral airways parameters}

Whether peripheral airways are a silent site of alterations in asthma or, rather, their involvement depicts a specific phenotype of the disease is a historical debate and carries potential clinical implications. A body of evidence has accumulated to confirm that, indeed, the pathophysiological changes of the peripheral airways can lead to excessive bronchoconstriction and, perhaps, more severe clinical manifestations, thus representing a specific "small airway phenotype". The issue remains as to what extent changes in functional parameters of small airways directly affect the clinical outcomes of patients with asthma [33, 34], since this can obviously affect the MCID.

The mean forced expiratory flow between $25 \%$ and $75 \%$ of $\mathrm{FVC}\left(\mathrm{FEF}_{25-75}\right)$ is one of the most popular indices of peripheral airways obstruction, resembling the concavity of the flow-volume curve [30]. However, its use in clinical practice is limited by issues of measurement inconsistency [35]. Serial measurements are indeed subjected to high variability and values are influenced by volume changes and obstruction of large airways. Assessing the MCID for this parameter is, therefore, a difficult task.

To the best of our knowledge, a precise MCID for lung function parameters reflecting small airway involvement has not been established. This is primarily due to the variable contribution of small airways changes in asthma with different levels of severity and magnitude of airway obstruction. Usmani et al. [36] estimated that, by reviewing all available studies, the prevalence of small airway functional change ranges between $50 \%$ and $60 \%$ in patients with asthma. Recently, POSTMA et al. [37] developed a specific score based on a combination of lung function measurements (impulse oscillometry variables, $\mathrm{FEF}_{50}$ and $\mathrm{FEF}_{25-75}$ both corrected for FVC and other parameters), and were able to detect small airways dysfunction. Although without a specific MCID threshold, the score showed a significant association with the duration of asthma, Asthma Control Questionnaire (ACQ)-6, number of exacerbations (positively), asthma control test (ACT) score, mini total Asthma Quality of Life Questionnaire, and EuroQol-5D-5L (negatively). The on-going longitudinal phase of the study will elucidate whether this score also predicts future changes in these clinical outcomes.

In the attempt to establish a MCID for small airway functional changes, several limitations need to be taken into account. These are mainly related to reproducibility, repeatability, acceptability, and variability of each test. These tests have never been employed as primary end-points in randomised controlled trials (RCTs), which lowers the evaluation of clinically important changes. The real challenge is to design clinical trials specifically targeting the small airways, proving the clinical importance of small airway treatment in asthmatics.

\section{Exercise tolerance}

Exercise tolerance can be defined as the ability to perform a physical task considered normal for a healthy person with the same characteristics. As it cannot confidently be predicted from variables such as $\mathrm{FEV}_{1}$, 
diffusing capacity of the lung for carbon monoxide and left ventricle ejection fraction, laboratory-based and field tests were developed. Assessment of exercise tolerance or capacity evaluation is rarely required in asthmatic patients, unless affected by fixed airway obstruction (functionally similar to COPD).

\section{Cardiopulmonary exercise testing and exercise challenge test}

Symptom-limited incremental cardiopulmonary exercise testing could be considered the gold standard for evaluating the causes of exercise intolerance. It is rarely required in asthmatic patients for clinical purposes, unless in the case of asthma with fixed airway obstruction (i.e. for pre-operatory evaluation) or for a differential diagnosis of dyspnoea [38].

A constant work-rate exercise test is the gold standard to study the effects of interventions on the endurance time and/or other measurable parameters. Again, it is rarely required in asthmatic patients for clinical purposes, except for cases of asthma with fixed airway obstruction or to measure the effect of a training programme.

Table 2 reports the MCID for symptom-limited incremental cardiopulmonary exercise testing and constant work-rate exercise test, derived from COPD populations [39].

Exercise challenge test on treadmill or cycloergometer is the gold standard to elicit exercise-induced bronchospasm (EIB). If the $\mathrm{FEV}_{1}$ decreases $\geqslant 10 \%$ at the end of exercise (up to $30 \mathrm{~min}$ after) the test is considered positive for EIB. Several RCTs have assessed the efficacy of pre-exercise use of bronchodilators in preventing EIB. Whilst complete avoidance of EIB is intuitively defined as a $\mathrm{FEV}_{1}$ decrease $<10 \%$ from baseline, protection from EIB has been suggested to be clinically meaningful if reduction in $\mathrm{FEV}_{1}$ decrease is $\geqslant 50 \%$ compared to the pre-treatment reference exercise challenge test [40].

Field tests: the six-minute walk test

Field tests require less technical equipment than laboratory-based tests. They are generally safe and cheaper but provide physicians with less physiological data.

The 6-min walk test is a self-paced test measuring the distance that a patient can quickly cover in a period of 6 min (6MWD), strongly related to important clinical outcomes. It is particularly used for measuring the response to medical interventions in patients with moderate-to-severe heart or lung disease [39, 41].

The 6MWD has a coefficient of variation of $\sim 8 \%$ in patients with COPD [42]. The effect of learning on the $6 \mathrm{MWD}$ is large enough to be clinically important (mean reported increase ranges from 0 to $17 \%$ ) and for this reason it has been suggested to perform two 6-min walk tests, with an interval of 15-20 min, and to record the best 6MWD [43].

Reference values for 6MWD are $\sim 580 \mathrm{~m}$ for healthy men and $500 \mathrm{~m}$ for healthy women [44]. Factors such as age, height, weight and sex should be taken into consideration when interpreting the results of single measurements made to determine functional status.

It initially proposed a MICD of $54 \mathrm{~m}$ in patients with COPD [45], but two documents recently concluded that the MCID lies between 25 and 33 m, independently of the disease [43, 46]. Most of the available data come from studies performed on patients affected by COPD before and after a rehabilitation programme rather than pharmacotherapy.

There is no clear evidence to suggest that MCID for 6MWD varies according to patient's characteristics. Hence, we can assume that these data are also reliable for patients with asthma, particularly in those with concomitant fixed airway obstruction.

$\begin{array}{ll}\text { TABLE } 2 \text { Suggested minimal clinically important difference (MCID) for cardiopulmonary } \\ \text { exercise testing outcomes } & \text { MCID } \\ \text { Protocol and measure } & 0.04 \pm 0.01 \mathrm{~L} \cdot \mathrm{min}^{-1} \\ & 4 \pm 1 \mathrm{~W} \\ \text { Symptom limited incremental exercise testing } & >0.14 \mathrm{~L} \text { or }>4.5 \% \text { predicted } \\ V_{\mathrm{O}_{2}} & \geqslant 2 \text { points } \\ \text { Work rate } & \geqslant 10-20 \mathrm{~mm} \\ \text { Inspiratory capacity } & \\ \text { Dyspnoea score on modified Borg scale (10 points) } & >100 \mathrm{~s} \text { or change }>33 \% \text { from baseline } \\ \text { Dyspnoea score on VAS (100 mm) } & \\ \text { Constant work rate exercise testing } & \end{array}$

Data refer to measurements at peak exercise. $V_{\mathrm{O}_{2}}$ : oxygen uptake; VAS: visual analogue scale. 


\section{Inflammatory biomarkers}

A precise approach to the patient with asthma, particularly with severe uncontrolled asthma, implies an evaluation of some parameters, or biomarkers, able to drive the clinician in tailoring the treatment. The role of biomarkers in asthma, particularly in severe uncontrolled asthma phenotype, and their correlation with clinical and "perceived" disease severity and response to the treatment is nowadays intensely investigated [47].

\section{Biomarkers in blood}

With regards to inflammatory biomarkers in blood, most current evidence focused on eosinophils, serum immunoglobulin (Ig)E and periostin. Recently, they have been widely investigated, in particular eosinophils, in the context of severe asthma as markers of disease severity and predictors of biologic treatment response.

To date, eosinophils are considered the most valuable biomarker of type- 2 inflammation in respiratory diseases, but their relevance in identifying asthma severity, as well as their correlation with specific clinical parameters (lung function, exhaled nitric oxide, exacerbation rate) and PROs (see Clinical end-points section) has not been widely investigated and it is not supported by univocal evidence [48-51]. However, blood eosinophilia seems to be more accurate and reliable as a marker of treatment response than asthma severity. However, despite blood eosinophilia being better related to the efficacy of omalizumab and anti-interleukin (IL)-5 drugs, no MCIDs in either clinical trials or real life have been specifically investigated or suggested by the authors. Similar conclusions can be described when evaluating the studies including other biologic drugs targeting eosinophils, i.e. reslizumab [52, 53] and benralizumab [54, 55].

In the specific case of omalizumab, since its first trial $\operatorname{IgE}$ antibodies have been investigated as a potential biomarker. Although this drug specifically targets IgE, it has been demonstrated that using total or allergen-specific levels of IgE could not be wholly effective to predict a therapeutic response [56]. This is the main reason why IgE could not be used as a biomarker and a MCID cannot be assessed.

Recently, serum periostin has been identified as a valuable biomarker of T-helper (Th)2 airways inflammation [57]. Its relevance as a biomarker for patient selection and predictor of response to traditional inhaled corticosteroids and Th2-targeted biologicals has been confirmed by a few other studies [58-61]. One of them has highlighted at each follow-up evaluation a point-by-point parallelism between serum periostin level and lung function, exhaled nitric oxide fraction $\left(F_{\mathrm{eNO}}\right)$ and PROs (i.e. ACT and Asthma Quality of Life Questionnaire); however, still no specific association with a MCID has been explored [60].

A number of other blood biomarkers have been explored by in vivo and in vitro studies (cytokines, chemokines, growth factors) [62], but their association with clinical parameters or treatment clinical effect has not been specifically addressed. Thus, to date MCID is also far from being identified.

\section{Biomarkers in exhaled air}

Very few data from studies analysing clinically important change of $F_{\mathrm{eNO}}$ in individual patients are available [61-67] and the results are different depending on the specific outcome. Data obtained from patients losing asthma control after steroid withdrawal showed a mean increase in $F_{\text {eNO }}$ ranging from $16 \mathrm{ppb}$ to $25 \mathrm{ppb}$, the latter representing a $60 \%$ increase from baseline [63, 64]. Comparable data came from the study of MichiLs et al. [65], where transition from well to poorly controlled asthma was associated with an increase in $F_{\mathrm{eNO}} \geqslant 40 \%$.

However, considering the change in $F_{\text {eNO }}$ during an acute event, the increase of values has been described as $50 \%$ higher in acute asthma attacks compared with when stability was restored [66], and up to $150 \mathrm{ppb}$ during exposure to a relevant allergen or acute infection $[67,68]$.

Considering the within-subject coefficient of variation, in healthy subjects this is $\sim 10 \%$ (corresponding to a raw change up to $4 \mathrm{ppb}$ ) [69, 70], while it increases to $20 \%$ in patients with asthma [69-71]; therefore, leading the American Thoracic Society to recommend a change of at least $20 \%$ to indicate a significant rise or fall in $F_{\mathrm{eNO}}$ over time or following an intervention [72].

\section{Biomarkers in sputum}

When used in clinical practice, the fold change and the absolute percentage change of inflammatory cells in the induced sputum are considered to have good statistical measurement properties and are recommended for use [73]. Notwithstanding that today the scientific community is very interested in the potential clinical value of MCID applied to asthma end-points, only a few contributions are available on the topic of induced sputum. 
In 2013, DASGUPTa et al. [74] first proposed a MCID equivalent to $15 \%$ absolute change in induced sputum eosinophils, which apparently is very high and may not be widely applicable in clinical practice. It also seems to conflict with other data linking a sputum eosinophil threshold of $>3 \%$ with a clinically important increase in exacerbation risk [73].

According to Demarche et al. [75], a MCID of 4.3\% in the percentage of sputum eosinophils (or 3.4-fold change) corresponds to a clinically meaningful deterioration in asthma control, measured by the ACQ score. We underline that these cut-offs were close to the two-fold change previously proposed by other authors and defined on the basis of the effect of corticosteroids [76, 77].

However, no study addressing the issue of MCID is available at the moment with regards to neutrophil count, IL-8, IL-13 and eosinophilic cationic protein in the induced sputum.

Overall, fragmentary and conflicting data on MCID on sputum eosinophils are available, mostly derived from studies assessing different end-points and performed in heterogeneous small populations. Multicentric studies on this topic that allow a definition and validation of a MCID threshold for eosinophils in sputum recognised by the scientific community are needed.

\section{Biomarkers in urine}

Currently, urinary biomarkers for asthma are not used in the common clinical practice and are usually evaluated for research purposes. Urinary leukotriene $\mathrm{E}_{4}$, bromotyrosine, eosinophil protein $\mathrm{X}$, club cell protein 16 and $9 \alpha-11 \beta$-prostaglandin F2 are the markers more widely evaluated in literature [78]. Still no specific association with a MCID cut-off has been explored or proposed.

\section{Clinical end-points: exacerbations and PROs}

\section{Symptoms}

Changes in asthmatic symptoms after active interventions can be measured by validated symptom scales that evaluate patient-reported symptoms [79].

An important issue arising from clinical trials is represented by the lack of convincing relationships between patient's subjective feelings and objective outcome measures. Indeed, the distributions of average minimal patient perceivable improvement and minimal patient perceivable deterioration scores often do not correlate with values of $\mathrm{FEV}_{1}$, peak expiratory flow and $F_{\mathrm{eNO}}[80]$.

Another limiting factor is represented by the possible influence of external features, like age and ongoing pharmacological treatments, on patient's perception of clinical improvement, which are often not considered by the measuring instruments currently available. For instance, small changes in asthma control measures correspond to higher levels of perceivable improvement in elderly than younger patients [79]. Although the average variation in asthma parameters shows an orderly progression in the expected direction with the categories of change in the global ratings, considerable variability exists among individuals. Indeed, individual patients perceive symptoms differently and hence also perceive the magnitude of change in their asthma differently when answering the global change [81-83]. Statistically significant differences from placebo could not be sufficient to demonstrate clinical improvement or decrease, if the clinical meaning of the difference is not understood, as already opportunely pointed out by JunIPEr [84].

Nowadays, clinical research in asthma lacks appropriate outcome standardisation [85]. As a consequence, the ability to examine and compare outcomes across clinical trials and studies, interpret evaluations of new and available therapeutic opportunities for asthma at a scale larger than single trial and pool data for observational studies is impaired [86]. Furthermore, the majority of published studies rarely indicate whether a study collected information on asthma symptoms, how this information was collected or how the information was used. For all these reasons, several national institutes that support asthma research have agreed to an effort for outcomes standardisation. In the context of this effort, expert subcommittees were established to propose and identify outcomes under three categories: 1) core, 2) supplemental, and 3) emerging [85].

For a complete standardisation and the diffusion of valid instruments for the measurement of asthma symptoms, these tools should be as effective as possible and should take into account the following methodological aspects: validity; internal consistency; test-retest reliability; and responsiveness or sensitivity to change. Examples of validated instruments for measuring asthma symptoms are shown in tables 3 and 4 [87-93].

\section{Exacerbations}

No validated MCID in reduction of severe asthma exacerbations is available. Exacerbations negatively impact on patients' quality of life and are potentially life-threatening events and the prevention of even a 
TABLE 3 Validated daily diaries and questionnaires for measurement of control of asthma symptoms

\begin{tabular}{l} 
Test \\
\hline $\begin{array}{l}\text { Pediatric Asthma } \\
\text { Caregiver Diary }\end{array}$ \\
\\
Daytime Symptom \\
Diary Scale and \\
Nocturnal Diary \\
Scale
\end{tabular}
Asthma Symptom
Utility Index
Adult patients

Adult patients

Adult patients

It has been also fully validated for children aged $>6$ years: $11-17$ years self-administered adult version; 6-10 years interviewer-administered version; it has not been validated in children aged $<6$ years old

\section{Question}

Asthma Control Test
Childhood Asthma Control Test
Children/caregivers of 4-11 year-old children

Measure

Report

- Mean daytime and night-time symptoms

- daytime symptoms: activity limitation, need for urgent care, SABA use $(7$ items; from 0 to 5-point scale);

- night-time symptoms: impact on caregiver, SABA use 13 items; from 0 to 5 -point scale)

Daily diary completed by adult patients:

- daytime symptoms (4 items; from 0 to 6-point scale);

- nocturnal awakening (1 item; from 0 to 3-point scale)

Retrospective questionnaire on previous 2 weeks:

- 11 items divided accordingly by the frequency and severity of asthma symptoms ( 8 items) and side-effects ( 3 items)

Retrospective questionnaire to measure the adequacy of asthma control and change in asthma control which occurs either spontaneously or as a result of treatment,

7 items divided according to the frequency and severity of daytime and night-time asthma symptoms (5 items), SABA use (1 item) and $\mathrm{FEV}_{1}$ (1 item)

Monthly retrospective questionnaire:

- Asthma composite score of 5 items on symptoms and daily functioning

- From 1 to 5-point scale lfor symptoms and activities: $1=$ all the time, to $5=$ not at all; for asthma control rating: $1=$ not controlled at all, to $5=$ completely controlled)

Monthly retrospective questionnaire:

- Asthma composite score of 7 items (1-4: children; 5-7: caregivers) on symptoms and daily functioning

- From 0 to 3-point scale for items $1-4$ and from 0 to 5 -point scale for items 5-7
- Mean scores /daily, weekly]

- Change over time is the difference between mean score from a 2-week baseline and a 2-week subsequent period

- Scoring uses a table for converting patient reported data into utilities (range 0-1)

- Mean scores

- Change in mean scores

Scoring uses a table for converting patient reported data into utilities

- Score range between 0 (totally controlled) and 6 (severely uncontrolled)

- Change in mean scores

- Score range from 5 (poor control) to 25 (complete controll

- Asthma Control Test score $>19$ indicates well-controlled asthma

- Score range 0-19: asthma symptoms not controlled

- Score range 20-27: well-controlled asthma
For adults: 3 points For patients aged 1218 years: 2 points

Not established

0.09 points

0.5 points

2 points

MCID: minimal clinically important difference; SABA: short-acting $\beta$-agonist; FEV1: forced expiratory volume in $1 \mathrm{~s}$. 
TABLE 4 Test-retest reliability, internal consistency and validity of some of the asthma tools used for measuring and reporting asthma symptoms/asthma control

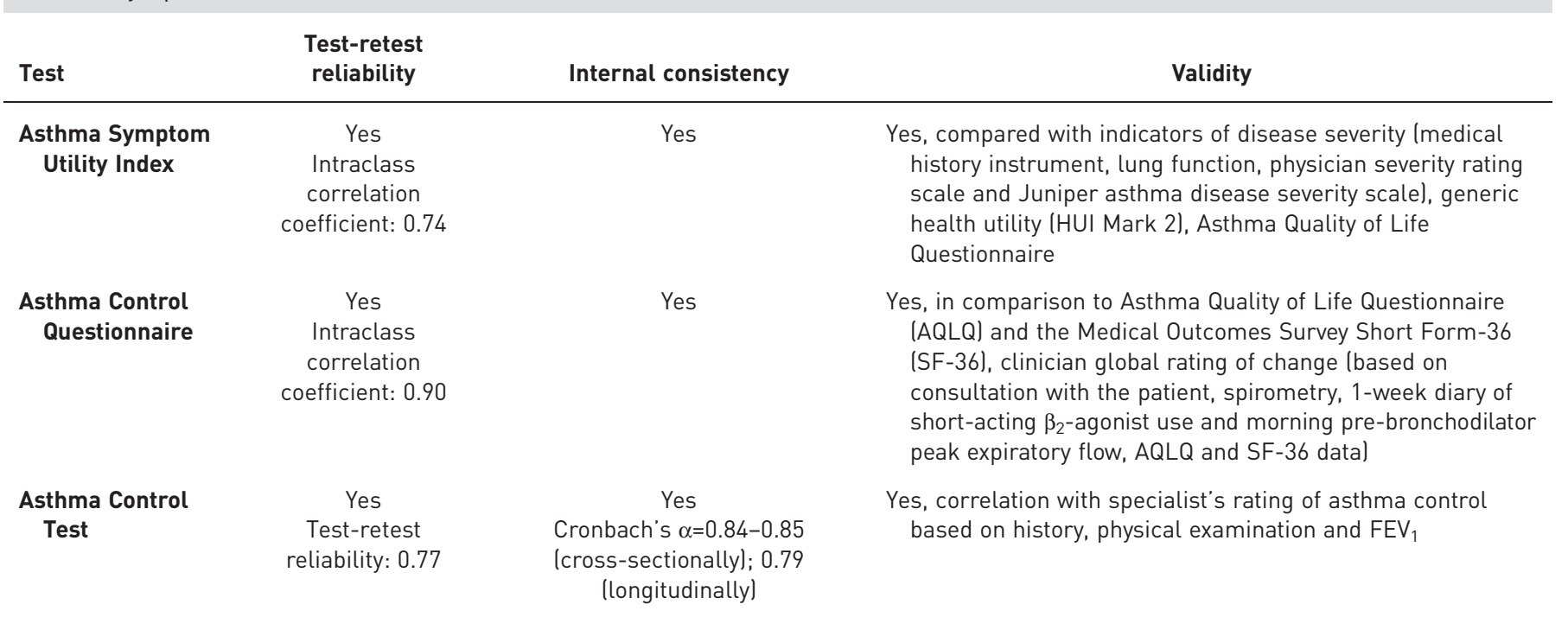

FEV1: forced expiratory volume in $1 \mathrm{~s}$.

single episode of severe exacerbation can be considered clinically relevant. A reduction in annual exacerbation rate or in the risk of having a severe asthma-related event ranging from $20-40 \%$ for a given asthma treatment regimen and/or intervention is considered clinically relevant in RCTs [94-98].

The development of novel digital tools able to explore and collect PROs [99] will give the opportunity to evaluate, in a standardised manner, the effects of asthma treatments not only on the frequency of exacerbations but also on the severity and time-course (i.e. duration and recovery) of the event.

\section{Questionnaires}

The assessment of PROs, such as disease control or health-related quality of life, increases clinical relevance for researchers and medical practitioners if the change related to a therapeutic intervention is perceived as clinically meaningful by the patient. Daily diaries and questionnaires represent the most commonly used tools in clinical practice and research studies to assess patient-centred outcomes.

MCIDs thresholds are available for several of the available questionnaires focusing on asthma symptoms (table 3).

The MCID of the Asthma Symptom Utility Index, which is a tool developed to assess the frequency and severity of asthma symptoms [89], was determined using anchor- and distribution-based methods. It has been defined in $\sim 0.09$ points, as it ranged from 0.07 to 0.09 in different evaluations according to the features of the considered population [90].

MCID for ACT [92] in an adult population was investigated through the distribution- and anchor-based methods [100]. The relationship of differences in ACT scores to the following items were evaluated: self-evaluated asthma severity; asthma exacerbation frequency in the previous 4 weeks; physician evaluated asthma control; physician recommendation of a change in therapy; $\left.\mathrm{FEV}_{1}\right)$; - the risk over the following 12 months of excess short-acting $\beta$-agonist use and exacerbations; and patient-reported changes in asthma course over 3 months. The analysis supported a MCID of 3 points, whereas in children from the age of 12 years (ACT) and 4-11 years (childhood ACT) MCID 1.9 (95\% CI 1.3-2.5) and 1.6 (95\% CI 1.1-2.1) points, was respectively [101]. Besides the different age groups included in the analysis, the selected methodology for calculating the MCID may account for the gap between the adult and paediatric studies. In fact the latter based the analysis on changes in quality of life instead of distribution- and anchor-based methods.

JUNIPER et al. [91] explored the measurement properties of three shortened versions of the ACQ composed by symptoms alone, symptoms plus $\mathrm{FEV}_{1}$ and symptoms plus short-acting $\beta$-agonist use, respectively. The identification of the MCID was based on minimal important changes in asthma-related quality of life. The results showed that the MCID for all versions was close to 0.5. Starting from these results the authors concluded that shortened versions of the ACQ can be used in large clinical trials without loss of validity or 
change in interpretation compared to the original questionnaire [102]. Interestingly, mean MCID in children aged 6-16 years old has also been shown to be similar to that observed in adults: $0.52 \pm 0.45$ points [103].

Apart from age, other parameters could influence the MCID and additional issues in evaluating PROs should be considered. Some researchers, for example, showed that using the overall score alone on currently available asthma questionnaires may not detect sex-specific differences in asthma symptoms, allowing asthma care providers to miss potential opportunities to develop targeted asthma care plans which may improve asthma control for their patients [104]. Also, disease severity can impact the MCID value. The example of the St George's Respiratory Questionnaire, a validated tool commonly used for measuring health-related quality of life in patients with COPD, is clear in this regard. Its established MCID corresponds to a decrease of $\geqslant 4$ units [105]; this threshold was similar in different studies regardless of the assessment methods, including expert and patient preference-based estimates and anchor-based analysis. However, this threshold was calculated from a population that did not include patients with severe disease. Thus, a different MCID was estimated by WeLLING et al. [106] for patients with severe COPD, through anchor- and distribution-based methods. MCID corresponded to 8.3 units and 7.1 units for 1- and 6-month follow-up after intervention, respectively.

Finally, even the route of administration of tools to assess PROs should be considered. Online self-management programmes for asthma have recently become available. International guidelines suggest that the ACQ can be used in these initiatives [4]. Nevertheless, the results might differ between different types of administration of the questionnaire. Results from a cross sectional study show significant difference between an online and an interviewer version of ACQ. In fact, a better control of asthma is perceived by patients when interacting with a caregiver than by online self-assessment [107].

In summary, MCID refers to the impact of an intervention from the patient's perspective and its evaluation should always be reported in clinical research assessing PROs. Since the MCID value may differ by disease severity, patient features and clinical context, the score value derived by validation studies should be considered but the MCID of the population exposed to specific therapeutic interventions should be calculated case by case [108].

\section{Conclusions and perspectives}

In a time in which scientific research is leading to a huge progress in the knowledge of pathophysiology of asthma, detailed definition of clinical phenotypes and endotypes and concrete developments in personalised medicine, it is mandatory not to lose sight of the most important aim in clinical practice: patient's well-being and quality of life. As underlined in this document, the concept of MCID returns to scientific research a dimension focused on clinical, rather than simply statistical goals and, therefore, represents a crucial element to guarantee the correct application of results coming from research studies in daily clinical activities. This seems to be particularly relevant in the context of a peculiar disease such as asthma, characterised by an extreme clinical variability in both the short and long term. Having measurable thresholds that are able to precisely monitor disease control or to confirm the efficacy of a specific treatment intervention is fundamental in dealing with asthma-related acute and chronic challenges.

Nevertheless, several limiting points about definition and use of MCID in real-life practice still need to be fully elucidated. Diverse methods of determination (i.e. Delphi versus distribution-based versus anchor-based) and dependence from modifiable (i.e. severity of disease, ongoing treatment, complexity of comorbidities) and non-modifiable (i.e. sex and age) patient's features make MCID a complex index to manage in view of its intrinsic limits of applicability to different clinical scenarios from the ones which led to its calculation. Therefore, it is essential for clinical researchers to standardise, as much as possible, the methodology that leads to the definition of MCID, taking into account all of the many facets that could affect its real clinical utility. In fact, regardless of the way of quantifying a clinically significant variation, sample and measurement errors may limit the accuracy of the established MCID. For that reason, when applying an MCID in clinical practice the overall body of available evidence rather than the result of one single methodology should be taken into consideration; furthermore, MCID interpretation and consequently its relevance in the patients' management should take into account differences and similarities between the study population for MCID identification and the patient being evaluated.

In addition, in the context of clinical trials, the comparative analysis of MCID outcomes needs to include that variability and requires appropriate statistical tests. In fact a MCID derived at the individual level may not be considered clinically important for comparison between treatment groups.

Moreover, future RCTs will have to not only include MCID among their outcomes, but also establish different MCIDs to target specific subgroups of patients. 
This document provides a series of MCIDs currently available in the literature and validated among populations of asthmatic patients, with most of the evidence focusing on outcomes as PROs, lung function and exercise tolerance. However, only scant and partial data are available for inflammatory biomarkers. These clearly represent the most interesting target for future development in the diagnosis and clinical management of asthma, particularly in view of the several biologic drugs in the pipeline, for which regulatory agencies will soon require personalised proof of efficacy and treatment response predictors.

Author contributions: All authors actively participated in determining the contents included in the review, drafting the manuscript and revising it critically. All authors approved the final version of the article for submission.

Conflict of interest: M. Bonini has nothing to disclose. M. Di Paolo has nothing to disclose. D. Bagnasco has nothing to disclose. I. Baiardini has nothing to disclose. F. Braido has nothing to disclose. M. Caminati has nothing to disclose. E. Carpagnano has nothing to disclose. M. Contoli reports grants from Chiesi, personal fees from Chiesi, AstraZeneca, Boehringer Ingelheim, Novartis Menarini, Mundipharma, Almirall and Zambon, and grants from University of Ferrara (Italy), outside the submitted work. A. Corsico has nothing to disclose. S. Del Giacco has nothing to disclose. E. Heffler has nothing to disclose. C. Lombardi has nothing to disclose. I. Menichini has nothing to disclose. M. Milanese has nothing to disclose. N. Scichilone has nothing to disclose. G. Senna has nothing to disclose. G.W. Canonica has nothing to disclose.

Support statement: The current work has been supported by a RESPIRE2 ERS/Marie-Curie Fellowship awarded to $\mathrm{M}$. Bonini and has been developed as a joint initiative on behalf of the Asthma Section of the Italian Society of Allergy, Asthma and Clinical Immunology (SIAAIC) and the Italian Respiratory Society (IRS).

\section{References}

1 Vos T, Flaxman AD, Naghavi M, et al. Years lived with disability (YLDs) for 1160 sequelae of 289 diseases and injuries 1990-2010: a systematic analysis for the Global Burden of Disease Study 2010. Lancet 2012; 380: 2163-2196.

2 Masoli M, Fabian D, Holt S, et al. The global burden of asthma: executive summary of the GINA Dissemination Committee report. Allergy 2004; 59: 469-478.

3 Nunes C, Pereira AM, Morais-Almeida M. Asthma costs and social impact. Asthma Res Pract 2017; 3: 1.

4 Global Initiative for Asthma. Global Strategy for Asthma Management and Prevention, 2018. www.ginasthma.org Date last accessed: 17 May 2018.

5 National Institute for Health and Care Excellence. Asthma: diagnosis, monitoring and chronic asthma management, 2017. www.nice.org.uk/guidance/ng80 Date last accessed: 17 May 172018.

6 British Thoracic Society/Scottish Intercollegiate Guidelines Network. British guideline on the management of asthma. A national clinical guideline, 2016. www.brit-thoracic.org.uk/document-library/clinical-information/ asthma/btssign-asthma-guideline-2016 Date last accessed: 17 May 2018.

$7 \quad$ Papi A, Brightling C, Pedersen SE, et al. Asthma. Lancet 2018; 391: 783-800.

8 Jaeschke R, Singer J, Guyatt GH. Measurement of health status. Ascertaining the minimal clinically important difference. Control Clin Trials 1989; 10: 407-415.

9 Lydick E, Epstein RS. Interpretation of quality of life changes. Qual Life Res 1993; 2: 221-226.

10 Wells G, Beaton D, Shea B, et al. Minimal clinically important differences: review of methods. J Rheumatol 2001; 28: 406-412.

11 Cook CE. Clinimetrics corner: the minimal clinically important change score (MCID): a necessary pretense. J Man Manip Ther 2008; 16: E82-E83.

12 Gatchel RJ, Lurie JD, Mayer TG. Minimal clinically important difference. Spine 2010; 35: 1739-1743.

13 McGlothlin AE, Lewis RJ. Minimal clinically important difference: defining what really matters to patients. JAMA 2014; 312: 1342-1343.

14 Wright A, Hannon J, Hegedus EJ, et al. Clinimetrics corner: a closer look at the minimal clinically important difference (MCID). J Man Manip Ther 2012; 20: 160-166.

15 Copay AG, Subach BR, Glassman SD, et al. Understanding the minimum clinically important difference: a review of concepts and methods. Spine J 2007; 7: 541-546.

16 Cohen J. Statistical power analysis for the behavioural sciences. New York, Academic Press, 1977.

17 Wyrwich KW, Bullinger M, Aaronson N, et al. Estimating clinically significant differences in quality of life outcomes. Qual Life Res 2005; 14: 285-295.

18 Norman GR, Sloan JA, Wyrwich KW. Interpretation of changes in health-related quality of life: the remarkable universality of half a standard deviation. Med Care 2003; 41: 582-592.

19 Katz P, Morris A, Trupin L, et al. Disability in valued life activities among individuals with systemic lupus erythematosus. Arthritis Rheum 2008; 59: 465-473.

20 Lu WS, Wang $\mathrm{CH}$, Lin $\mathrm{JH}$, et al. The minimal detectable change of the simplified stroke rehabilitation assessment of movement measure. J Rehabil Med 2008; 40: 615-619.

21 Leopold SS, Porcher R. The minimum clinically important difference - the least we can do. Clin Orthop Relat Res 2017; 475: 929-932.

22 Wyrwich KW, Wolinsky FD. Identifying meaningful intra-individual change standards for health-related quality of life measures. J Eval Clin Pract 2000; 6: 39-49.

23 Turner D, Schünemann HJ, Griffith LE, et al. Using the entire cohort in the receiver operating characteristic analysis maximizes precision of the minimal important difference. J Clin Epidemiol 2009; 62: 374-379.

24 Crosby RD, Kolotkin RL, Williams GR. An integrated method to determine meaningful changes in health-related quality of life. J Clin Epidemiol 2004; 57: 1153-1160.

25 Kuzma JW, Bohnenblust S. Basic Statistics for the Health Science. 5th Edn. New York, McGraw-Hill Education, 2005. 
Revicki D, Hays $\mathrm{RD}$, Cella $\mathrm{D}$, et al. Recommended methods for determining responsiveness and minimally important differences for patient-reported outcomes. J Clin Epidemiol 2008; 61: 102-109.

Committee for Proprietary Medicinal Products. Note for guidance on the clinical investigation of medicinal products in the treatment of asthma. London, CPMP/EWP/2922/01, 2002.

Pennock BE, Rogers RM, McCaffree DR. Changes in measured spirometric indices. What is significant? Chest 1981; 80: 97-99.

Wise RA, Connett J, Kurnow K, et al. Selection of spirometric measurements in a clinical trial, the Lung Health Study. Am J Respir Crit Care Med 1995; 151: 675-681.

Pellegrino R, Viegi G, Brusasco V, et al. Interpretative strategies for lung function tests. Eur Respir J 2005; 26 : 948-968.

Cerveri I, Corsico AG, Accordini S, et al. What defines airflow obstruction in asthma? Eur Respir J 2009; 34 568-573.

Perez T, Chanez P, Dusser D, et al. Prevalence and reversibility of lung hyperinflation in adult asthmatics with poorly controlled disease or significant dyspnea. Allergy 2016; 71: 108-114.

a time for reappraisal. Allergy 2010; 65: 141-151.

Scichilone N, Battaglia S, Olivieri D, et al. The role of small airways in monitoring the response to asthma treatment: what is beyond FEV1? Allergy 2009; 64: 1563-1569.

Sorkness RL, Bleecker ER, Busse WW, et al. Lung function in adults with stable but severe asthma: air trapping and incomplete reversal of obstruction with bronchodilation. J Appl Physiol 2008; 104: 394-403.

Usmani OS, Singh D, Spinola M, et al. The prevalence of small airways disease in adult asthma: a systematic literature review. Respir Med 2016; 116: 19-27.

Postma DS, Brightling C, Baldi S, et al. Exploring the relevance and extent of small airways dysfunction in asthma (ATLANTIS): baseline data from a prospective cohort study. Lancet Respir Med 2019; 7: 402-416.

Palange P, Ward SA, Carlsen KH, et al. Recommendations on the use of exercise testing in clinical practice. Eur Respir J 2007; 29: 185-209.

Puente-Maestu L, Palange P, Casaburi R, et al. Use of exercise testing in the evaluation of interventional efficacy: an official ERS statement. Eur Respir J 2016; 47: 429-460.

Bonini M, Permaul P, Kulkarni T, et al. Loss of salmeterol bronchoprotection against exercise in relation to ADRB2 Arg16Gly polymorphism and exhaled nitric oxide. Am J Respir Crit Care Med 2013; 188: 1407-1412.

ATS Committee on Proficiency Standards for Clinical Pulmonary Function Laboratories. ATS statement: guidelines for the six-minute walk test. Am J Respir Crit Care Med 2002; 166: 111-117.

Solway S, Brooks D, Lacasse Y, et al. A qualitative systematic overview of the measurement properties of functional walk tests used in the cardiorespiratory domain. Chest 2001; 119: 256-270.

Holland AE, Spruit MA, Troosters T, et al. An official European Respiratory Society/American Thoracic Society technical standard: field walking tests in chronic respiratory disease. Eur Respir J 2014; 44: 1428-1446.

Enright PL, Sherrill DL. Reference equations for the six-minute walk in healthy adults. Am J Respir Crit Care Med 1998; 158: 1384-1387. minute walk test in chronic lung disease patients. Am J Respir Crit Care Med 1997; 155: 1278-1282.

Singh SJ, Puhan MA, Andrianopoulos V, et al. An official systematic review of the European Respiratory Society/ American Thoracic Society: measurement properties of field walking tests in chronic respiratory disease. Eur Respir J 2014; 44: 1447-1478.

Bagnasco D, Passalacqua G, Caminati M, et al. Evolving phenotypes to endotypes: is precision medicine achievable in asthma? Expert Rev Respir Med 2020; 14: 163-172.

Hastie AT, Moore WC, Li H, et al. Biomarker surrogates do not accurately predict sputum eosinophil and neutrophil percentages in asthmatic subjects. J Allergy Clin Immunol 2013; 132: 72-80.

Silkoff PE, Strambu I, Laviolette M, et al. Asthma characteristics and biomarkers from the Airways Disease Endotyping for Personalized Therapeutics (ADEPT) longitudinal profiling study. Respir Res 2015; 16: 142.

Price DB, Rigazio A, Campbell JD, et al. Blood eosinophil count and prospective annual asthma disease burden: a UK cohort study. Lancet Respir Med 2015; 3: 849-858.

Tran TN, Khatry DB, Ke X, et al. High blood eosinophil count is associated with more frequent asthma attacks in asthma patients. Ann Allergy Asthma Immunol 2014; 113: 19-24.

Bjermer L, Lemiere C, Maspero J, et al. Reslizumab for inadequately controlled asthma with elevated blood eosinophil levels: a randomized phase 3 study. Chest 2016; 150: 789-798.

Corren J, Weinstein S, Janka L, et al. Phase 3 study of reslizumab in patients with poorly controlled asthma: effects across a broad range of eosinophil counts. Chest 2016; 150: 799-810.

Bleecker ER, FitzGerald JM, Chanez P, et al. Efficacy and safety of benralizumab for patients with severe asthma uncontrolled with high-dosage inhaled corticosteroids and long-acting $\beta 2$-agonists (SIROCCO): a randomised, multicentre, placebo-controlled phase 3 trial. Lancet 2016; 388: 2115-2127.

FitzGerald JM, Bleecker ER, Nair P, et al. Benralizumab, an anti-interleukin-5 receptor $\alpha$ monoclonal antibody, as add-on treatment for patients with severe, uncontrolled, eosinophilic asthma (CALIMA): a randomised, double-blind, placebo-controlled phase 3 trial. Lancet 2016; 388: 2128-2141.

Wahn U, Martin C, Freeman P, et al. Relationship between pretreatment specific IgE and the response to omalizumab therapy. Allergy 2009; 64: 1780-1787.

Idolazzi L, Ridolo E, Fassio A, et al. Periostin: The bone and beyond. Eur J Intern Med 2017; 38: 12-16.

Izuhara K, Conway SJ, Moore BB, et al. Roles of periostin in respiratory disorders. Am J Respir Crit Care Med 2016; 193: 949-956.

Tajiri T, Matsumoto H, Gon Y, et al. Utility of serum periostin and free IgE levels in evaluating responsiveness to omalizumab in patients with severe asthma. Allergy 2016; 71: 1472-1479.

Caminati M, Gatti D, Dama A, et al. Serum periostin during omalizumab therapy in asthma: A tool for patient selection and treatment evaluation. Ann Allergy Asthma Immunol 2017; 119: 460-462.

Corren J, Lemanske RF, Hanania NA, et al. Lebrikizumab treatment in adults with asthma. N Engl J Med 2011; 365: 1088-1098. 
Richards LB, Neerincx AH, van Bragt JJMH, et al. Biomarkers and asthma management: analysis and potential applications. Curr Opin Allergy Clin Immunol 2018; 18: 96-108.

Beck-Ripp J, Griese M, Arenz S, et al. Changes of exhaled nitric oxide during steroid treatment of childhood asthma. Eur Respir J 2002; 19: 1015-1019.

Jones SL, Kittelson J, Cowan JO, et al. The predictive value of exhaled nitric oxide measurements in assessing changes in asthma control. Am J Respir Crit Care Med 2001; 164: 738-743.

Michils A, Baldassarre S, Van Muylem A. Exhaled nitric oxide and asthma control: a longitudinal study in unselected patients. Eur Respir J 2008; 31: 539-546.

Massaro AF, Gaston B, Kita D, et al. Expired nitric oxide levels during treatment of acute asthma. Am J Respir Crit Care Med 1995; 152: 800-803.

Rolla G, Malinovschi A, Badiu I, et al. The increase in exhaled NO following allergen challenge is not associated with airway acidification. Eur J Clin Invest 2011; 41: 411-416.

Ferrazzoni S, Scarpa MC, Guarnieri G, et al. Exhaled nitric oxide and breath condensate $\mathrm{pH}$ in asthmatic reactions induced by isocyanates. Chest 2009; 136: 155-162.

Buchvald F, Baraldi E, Carraro S, et al. Measurements of exhaled nitric oxide in healthy subjects age 4 to 17 years. J Allergy Clin Immunol 2005; 115: 1130-1136.

Ekroos H, Karjalainen J, Sarna S, et al. Short-term variability of exhaled nitric oxide in young male patients with mild asthma and in healthy subjects. Respir Med 2002; 96: 895-900.

Pijnenburg MW, Floor SE, Hop WC, et al. Daily ambulatory exhaled nitric oxide measurements in asthma. Pediatr Allergy Immunol 2006; 17: 189-193.

Dweik RA, Boggs PB, Erzurum SC, et al. An official ATS clinical practice guideline: interpretation of exhaled nitric oxide levels ( $\mathrm{F}_{\mathrm{ENO}}$ ) for clinical applications. Am J Respir Crit Care Med 2011; 184: 602-615.

Gibson PG. Why inflammatory phenotyping is necessary for successful drug evaluation in asthma and COPD. Eur Respir J 2013; 42: 891-892.

Dasgupta A, Zhang S, Thabane L, et al. Sample sizes for clinical trials using sputum eosinophils as a primary outcome. Eur Respir J 2013; 42: 1003-1011.

Demarche SF, Schleich FN, Paulus VA, et al. Asthma control and sputum eosinophils: a longitudinal study in daily practice. J Allergy Clin Immunol Pract 2017; 5: 1335-1343.

Reddel HK, Taylor DR, Bateman ED, et al. An official American Thoracic Society/European Respiratory Society statement: asthma control and exacerbations: standardizing endpoints for clinical asthma trials and clinical practice. Am J Respir Crit Care Med 2009; 180: 59-99.

Kips JC, Inman MD, Jayaram L, et al. The use of induced sputum in clinical trials. Eur Respir J 2002; 20: Suppl. 37, 47s-50s.

Szefler SJ, Wenzel S, Brown R, et al. Asthma outcomes: biomarkers. J Allergy Clin Immunol 2012; 129: Suppl. 3, S9-23.

Santanello NC, Zhang J, Seidenberg B, et al. What are minimal important changes for asthma measures in a clinical trial? Eur Respir J 1999; 14: 23-27.

Senna G, Passalacqua G, Schiappoli M, et al. Correlation among FEV, nitric oxide and asthma control test in newly diagnosed asthma. Allergy 2007; 62: 207-208.

Connolly MJ, Crowley JJ, Charan NB, et al. Reduced subjective awareness of bronchoconstriction provoked by methacholine in elderly asthmatic and normal subjects as measured on a simple awareness scale. Thorax 1992; 47: 410-413.

Rubinfeld AR, Pain MC. Conscious perception of bronchospasm as a protective phenomenon in asthma. Chest 1977; 72: 154-158.

Rubinfeld AR, Pain MC. Perception of asthma. Lancet 1976; 1: 882-884. Immunol 1998; 102: 16-17.

Krishnan JA, Lemanske RF Jr, Canino GJ, et al. Asthma outcomes: symptoms. J Allergy Clin Immunol 2012; 129: Suppl. 3, S124-S135.

Davis SQ, Permutt Z, Permutt S, et al. Perception of airflow obstruction in patients hospitalized for acute asthma. Ann Allergy Asthma Immunol 2009; 102: 455-461.

Santanello NC, Demuro-Mercon C, Davies G, et al. Validation of a pediatric asthma caregiver diary. J Allergy Clin Immunol 2000; 106: 861-866.

Santanello NC, Barber BL, Reiss TF, et al. Measurement characteristics of two asthma symptom diary scales for use in clinical trials. Eur Respir J 1997; 10: 646-651.

Revicki DA, Leidy NK, Brennan-Diemer F, et al. Integrating patient preferences into health outcomes assessment: the multiattribute Asthma Symptom Utility Index. Chest 1998; 114: 998-1007.

Bime C, Wei CY, Holbrook JT, et al. Asthma symptom utility index: reliability, validity, responsiveness, and the minimal important difference in adult asthmatic patients. J Allergy Clin Immunol 2012; 130: 1078-1084.

Juniper EF, O'Byrne PM, Guyatt GH, et al. Development and validation of a questionnaire to measure asthma control. Eur Respir J 1999; 14: 902-907.

Nathan RA, Sorkness CA, Kosinski M, et al. Development of the asthma control test: a survey for assessing asthma control. J Allergy Clin Immunol 2004; 113: 59-65.

Liu AH, Zeiger R, Sorkness C, et al. Development and cross-sectional validation of the Childhood Asthma Control Test. J Allergy Clin Immunol 2007; 119: 817-825.

Pauwels RA, Pedersen S, Busse WW, et al. Early intervention with budesonide in mild persistent asthma: a randomised, double-blind trial. Lancet 2003; 361: 1071-1076. asthma exacerbations: a randomised controlled, double-blind study. Lancet 2006; 368: 744-753.

Papi A, Corradi M, Pigeon-Francisco C, et al. Beclometasone-formoterol as maintenance and reliever treatment in patients with asthma: a double-blind, randomised controlled trial. Lancet Respir Med 2013; 1: 23-31.

Ortega HG, Liu MC, Pavord ID, et al. Mepolizumab treatment in patients with severe eosinophilic asthma. N Engl J Med 2014; 371: 1198-1207. 
Castro M, Wenzel SE, Bleecker ER, et al. Benralizumab, an anti-interleukin 5 receptor $\alpha$ monoclonal antibody, versus placebo for uncontrolled eosinophilic asthma: a phase $2 \mathrm{~b}$ randomised dose-ranging study. Lancet Respir Med 2014; 2: 879-890.

99 Singh D, Kampschulte J, Wedzicha JA, et al. A trial of beclomethasone/formoterol in COPD using EXACT-PRO to measure exacerbations. Eur Respir J 2013; 41: 12-17.

100 Schatz M, Kosinski M, Yarlas AS, et al. The minimally important difference of the Asthma Control Test. J Allergy Clin Immunol 2009; 124: 719-723.

101 Voorend-van Bergen S, Vaessen-Verberne AA, Landstra AM, et al. Monitoring childhood asthma: web-based diaries and the asthma control test. J Allergy Clin Immunol 2014; 133: 1599-605.

102 Juniper EF, Svensson K, Mörk AC, et al. Measurement properties and interpretation of three shortened versions of the asthma control questionnaire. Respir Med 2005; 99: 553-558.

103 Juniper EF, Gruffydd-Jones K, Ward S, et al. Asthma Control Questionnaire in children: validation, measurement properties, interpretation. Eur Respir J 2010; 36: 1410-1416.

104 McCallister JW, Holbrook JT, Wei CY, et al. Sex differences in asthma symptom profiles and control in the American Lung Association Asthma Clinical Research Centers. Respir Med 2013; 107: 1491-1500.

105 Jones PW. St. George's Respiratory Questionnaire: MCID. COPD 2005; 2: 75-79.

106 Welling JB, Hartman JE, Ten Hacken NH, et al. The minimal important difference for the St George's Respiratory Questionnaire in patients with severe COPD. Eur Respir J 2015; 46: 1598-1604.

107 Honkoop PJ, Loijmans RJ, Termeer EH, et al. Comparison between an online self-administered and an interviewer-administered version of the Asthma Control Questionnaire: a cross-sectional validation study. Prim Care Respir J 2013; 22: 284-289.

108 Revicki DA, Cella D, Hays RD, et al. Responsiveness and minimal important differences for patient reported outcomes. Health Qual Life Outcomes 2006; 4: 70. 